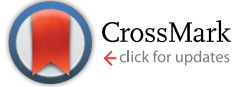

Cite this: RSC Adv., 2015, 5, 22906

Received 7th January 2015

Accepted 19th February 2015

DOI: $10.1039 / \mathrm{c} 5 \mathrm{ra00258c}$

www.rsc.org/advances

\section{Thermally controlled growth of carbon onions within porous graphitic carbon-detonation nanodiamond monolithic composites $\uparrow$}

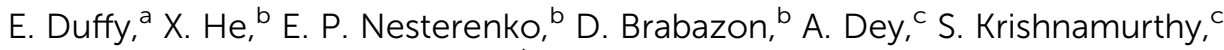 \\ P. N. Nesterenko a and B. Paull ${ }^{\star a d}$
}

Unique porous carbon monoliths containing thermally annealed carbon onions, were prepared from a resorcinol formaldehyde precursor rod, containing silica gel acting as a hard template, detonation nanodiamond, and $\mathrm{Fe}^{3+}$ as a graphitisation catalyst. Detonation nanodiamond was converted to carbon onions during controlled pyrolysis under $\mathrm{N}_{2}$, where the temperature cycle reached a maximum of 1250 ${ }^{\circ} \mathrm{C}$. Thermal characterisation and high resolution electron microscopy have confirmed the graphitisation of nanodiamond, and revealed the resulting quasi-spherical carbon onions with an average particle size of $5.24 \mathrm{~nm}$. The bimodal porous composite contains both macropores $(5 \mu \mathrm{m})$ and mesopores $(10 \mathrm{~nm})$, with a BET surface area of $214 \mathrm{~m}^{2} \mathrm{~g}^{-1}$ for a nanodiamond prepared monolith $(0.012$ wt\% nanodiamond in the precursor mixture), approximately twice that of blank monoliths, formed without the addition of nanodiamond, thus providing a new approach to increase surface area of such porous carbon rods. Raman spectroscopy and X-ray photoelectron spectroscopy also confirmed an enhanced graphitisation of the monolithic carbon skeleton resulting from the elevated thermal conductivity of the added nanodiamond. TEM imaging has confirmed the nanodiamond remains intact following pyrolysis at temperatures up to $900^{\circ} \mathrm{C}$.

\section{Introduction}

New porous materials continue to attract significant interest within the science and technology community due to their unique and versatile properties, and advanced applications. ${ }^{1}$ In particular, the design of porous carbon materials with tailored chemical and structural properties has immense significance within the fabrication, coatings and coating and energy storage based industries, addressing key technological challenges. For porous carbons, these properties include high specific surface area, chemical inertness, thermal stability and electronic conductivity. The use of porous carbons as an electrode material $^{2}$ and indeed in electrochemical double layer capacitors, or super capacitors ${ }^{3}$ is therefore currently very topical. In the area of environmental technology, there are reports demonstrating

\footnotetext{
${ }^{a}$ Australian Centre for Research on Separation Science, School of Physical Sciences, University of Tasmania, Hobart, Tasmania, Australia.E-mail: brett.paull@utas.edu.au ${ }^{b}$ Irish Separation Science Cluster, National Centre for Sensor Research, Dublin City University, Dublin 9, Ireland

${ }^{c}$ Department of Engineering and Innovation, The Open University, Milton Keynes MK 7 $6 A A, U K$

${ }^{d} A R C$ Centre of Excellence for Electromaterials Science, School of Physical Sciences, University of Tasmania, Hobart, Tasmania, Australia

$\uparrow$ Electronic supplementary information (ESI) available. See DOI: $10.1039 / \mathrm{c} 5 \mathrm{ra00258c}$
}

utility as catalyst supports, e.g. for gas separation or storage, ${ }^{\mathbf{4 , 5}}$ and as adsorbents for separation or remediation processes. ${ }^{6}$

Several excellent reviews on the subject of porous carbon production and applications have recently emerged..$^{7-9}$ One novel route to the production of porous carbon materials is the formation of so-called carbon monoliths. These typically exhibit a hierarchical porous structure, and are often produced through various templating methods, for example the use of hard and soft removable templates. Silica particles (including mesoporous silica or silica nanoparticles) are commonly used in the hardtemplating synthesis of porous carbon monoliths, to facilitate creation of a controlled macroporous structure in the final material. The inorganic template is embedded in the carbon precursor, or the carbon precursor is introduced into the pores of the template. After undergoing carbonisation via pyrolysis, the template is removed, thus generating a porous material with isolated pores or an interconnected pore network. Mesopores may also be created by catalytic graphitisation and this is commonly achieved using a metal ion catalyst, or by the carbonisation of a polymer blend containing a carbon precursor polymer and a decomposable polymer that is removed to produce the pores. Reports on the production of such hierarchical porous carbons with bimodal pore structures have been published, ${ }^{7-10}$ several of which are based on the polymerisation and carbonisation of silica particle embedded resorcinol formaldehyde (RF) resin, using $\mathrm{Fe}^{3+}$ as a catalyst to enhance graphitisation. ${ }^{\mathbf{1 0 , 1 1}}$ 
Porous carbon monoliths offer inter-connected channels within their pore network, with a high flow-through permeability, good thermal and electrical conductivity hence their potential application in adsorption or separation processes, ${ }^{10-12}$ and electrochemical energy storage applications. ${ }^{13}$ Recently, the use of nanocarbons to form porous carbon monoliths or carbon-carbon composite materials has produced some exciting results, showing considerably enhanced electrochemical properties for potential applications in the above areas. ${ }^{14,15}$ Indeed, changes to the physical, chemical and electrochemical properties of the nano-composite monoliths have been reported, when compared to simple carbon monoliths without embedded or surface exposed nanocarbons. Physical enhancements include higher specific surface area, greater mesopore volume and a narrower pore size distribution. ${ }^{16}$ However, to-date such studies are limited in number, and controlled approaches to achieve substantially enhanced properties, e.g. surface area, for such monolithic substrates are rather limited.

It can be expected that with the inclusion of nanocarbons within porous carbon monoliths, there will be a transfer of unique physical-chemical properties to the final composite, provided the nanocarbon is preserved through any carbonisation process. These unique nanocarbon properties have seen them applied in a wide variety of ways in recent times, e.g. in environmental applications as sensors, filters, and sorbents, ${ }^{17}$ with fullerenes, carbon nanotubes and carbon onions having demonstrated high sorption capacities for organic pollutants and heavy metal contaminants. ${ }^{18}$ Recently, nanodiamond (ND) has received renewed attention, primarily due to its biocompatibility and potential applications in drug delivery. ${ }^{19,20}$ Nanoscale diamond $\left(\mathrm{sp}^{3}\right.$ carbon) may be produced by detonation synthesis, where the detonation soot is purified under oxidative acidic conditions to yield detonation nanodiamond (DND), which is known to have a high thermal conductivity, mechanical stability and surface chemistry readily amenable to functionalisation. Carbon onions may be produced by the thermal annealing of $\mathrm{ND}^{21}$ in an inert atmosphere or under vacuum. They too have a variety of interesting properties (such as high surface area and electrical conductivity), making them of interest for use in supercapacitors, ${ }^{22}$ lubrication, and in environmental remediation. ${ }^{18}$

Therefore, herein is described a novel route for the preparation and characterisation of a new porous carbon monolith with significantly enhanced surface area and graphitic character, via embedded carbon onions produced through the controlled thermal annealing of DND during the carbonisation process. The new carbon on carbon composite material was formed by pyrolysis of a precursor rod containing a mixture of RF resin, silica particles and DND, with a $\mathrm{Fe}^{3+}$ catalyst used to increase localised graphitisation. The thermal conductivity of the DND was also expected to enhance the graphitisation process, and the precise thermal conversion of diamond particles to carbon onions during the pyrolysis (to a maximum temperature of $1250{ }^{\circ} \mathrm{C}$ ) was explored.

\section{Experimental}

\section{Synthesis of graphitic carbon monoliths with thermally annealed carbon onions}

Fig. 1(A) to 1(C) illustrates the steps taken in preparing the nanocomposite porous graphitic carbon monoliths, and a blank carbon monolithic material (containing no additional DND). The precursor solution (A) is composed of a polymeric mixture containing a RF resin in 1-butanol. In preparation of this solution, $1 \mathrm{~g}$ of $5 \mu \mathrm{m}$ silica particles, with a surface area of $359 \mathrm{~m}^{2} \mathrm{~g}^{-1}$ and a pore size of $550 \AA$, (Nucleosil silica beads from Macherey-Nagel, Duren, Germany) was dispersed in $1.85 \mathrm{~mL}$ 1-butanol and sonicated for 1 hour before adding $0.18 \mathrm{~g}$ of ferric chloride (99\% Riedel-De Haen, Seelze, Hannover, Germany) and $0.367 \mathrm{~g}$ of resorcinol (99\% Sigma-Aldrich, Dublin, Ireland). A $555 \mu \mathrm{L}$ aliquot of aqueous DND suspension (Single Digit Nanodiamond, $50 \mathrm{mg}$ $\mathrm{mL}^{-1}$ aqueous suspension, PlasmaChem GmbH Germany) was added to this RF polymeric resin, as a percentage $(30 \%)$ of the total volume of 1-butanol present. The addition of $0.3 \mathrm{~g}$ of icecooled formaldehyde (37 wt\% solution, Sigma-Aldrich, Dublin, Ireland) to the mixture was made, with constant stirring. Following stirring in an ice-bath for 1 hour, the resin was transferred to a $7 \mathrm{~mm}$ i.d. glass tube, which was capped and sealed for polymerisation at $90{ }^{\circ} \mathrm{C}$ in a water bath (GFL water bath from Laborggerateborse $\mathrm{GmbH}$, Burladingen, Germany) for 15 hours. A solid rod was formed, which was detached slightly from the walls of the tube due to shrinkage during polymerisation. A drying step (72 hours in the fume hood) to allow the slow evaporation of any remaining solvent was carried out, and materials were then dried thoroughly in a vacuum oven to ensure the removal of any remaining solvents (EHRET vacuum oven from Ehret Labor and Pharmatechnik GmbH, KG, Emmendingen, Germany). Pyrolysis of the materials was carried out in a horizontal tube furnace (model GSL1300X from MTI, Richmond, VA, U.S.A), which was purged with nitrogen. The full temperature program applied involved an initial ramp from room temperature to $800^{\circ} \mathrm{C}$ at a rate of $2.5^{\circ} \mathrm{C} \mathrm{min}^{-1}$. The temperature was held at $800{ }^{\circ} \mathrm{C}$ for 2 hours, and then further increased to either $900{ }^{\circ} \mathrm{C}$ or $1250{ }^{\circ} \mathrm{C}$ at a rate of $10^{\circ} \mathrm{C} \min ^{-1}$. The maximum temperature was held for 1 hour, and the furnace was subsequently allowed to cool naturally to room temperature. The carbon rods obtained showed an irregular mesoporous structure by SEM imaging (as discussed below), with the silica particles remaining intact and carbon onions present resulting from the thermal annealing of the DND $\left(1250{ }^{\circ} \mathrm{C}\right)$. The rods then underwent hydrofluoric acid etching in order to remove the silica template and the Fe catalyst. The HF acid (38-40\% Sigma-Aldrich, Dublin, Ireland) etching step involved submersion of the carbon rods for 5 hours, followed by washing with copious amounts of deionised water until a neutral $\mathrm{pH}$ was attained. Finally, the rods were dried in the vacuum oven at $80{ }^{\circ} \mathrm{C}$ for 16 hours and an example of the final material obtained is shown in Fig. 1(D).

\section{Material characterisation}

High-resolution images of the porous carbon materials were taken using Transmission Electron Microscopy (TEM), JEOL 

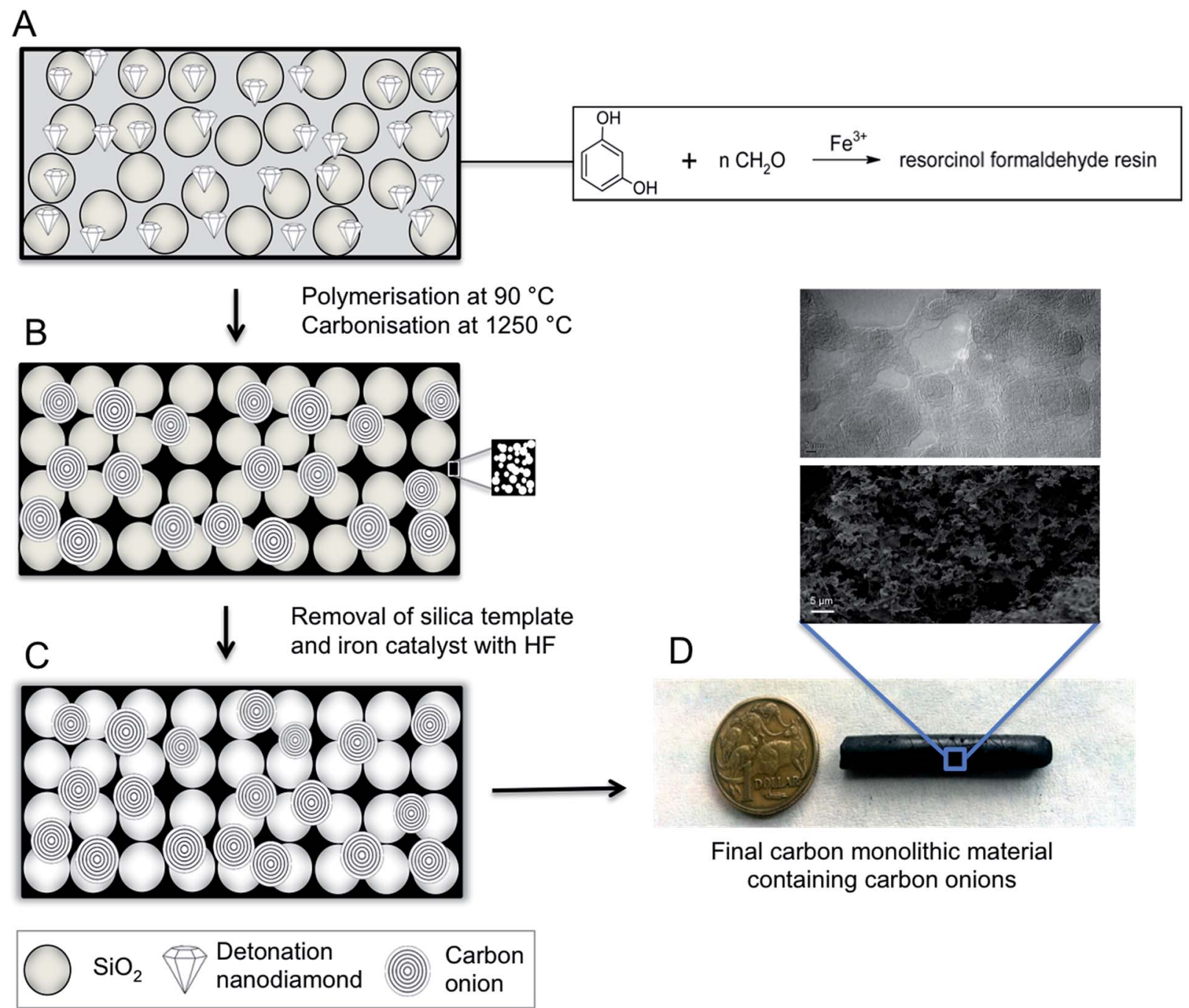

Fig. 1 Scheme for fabrication and pyrolysis of carbon monolithic composites.

model JEM 2100, equipped with an Orius camera in-line (Gatan, Pleasenton, CA, U.S.A). A lanthanum hexaboride filament was used at an accelerating voltage of $200 \mu \mathrm{A}$. TEMCON software was used to control the instrument, and Digital Micrograph software (Gatan) was used in controlling the camera. The sample holder used was the type EM21010 single tilt holder and the preparation of samples involved the sonication of a small fragment of porous carbon monolithic material in $1 \mathrm{~mL}$ of isopropanol in an Eppendorf tube, until a dilute suspension of the solid was formed. ${ }^{23}$ Then a $1 \mathrm{~mL}$ fine-tipped plastic pipette was used to place one drop of the suspension on to a S-160-3 carbon film mesh $\mathrm{Cu}$ (50) (Agar Scientific) for imaging. Samples were stored in plastic petri-dishes on filter paper to allow evaporation of the solvent prior to imaging. Scanning Electron Microscopy (SEM) imaging was performed on a Hitachi SU70 instrument (Hitachi High Technologies America, USA), and sample preparation involved placing a small cross section of the porous carbon materials on to carbon tape on an Al SEM stub. Samples were sputter coated with a thin $(\sim 4 \mathrm{~nm})$ layer of platinum prior to imaging at $1.5 \mathrm{kV}$. Energy dispersive X-ray electron spectroscopy (EDX) was subsequently carried out on the same samples, where an excitation energy of $4 \mathrm{kV}$ was used. Specific surface areas and pore volumes were measured using a surface area analyser (model TriStar II 3020, Micromeritics Gemini, Georgia, USA) through the nitrogen adsorption-desorption technique. Prior to measurement, crushed samples were dried overnight, at $100{ }^{\circ} \mathrm{C}$, under vacuum. Raman spectra were measured on a LabRam800HR instrument (Horiba Jobin Yvon, Northampton, U.K). An argon laser (Innova 70-C-2 from Coherent, Santa Clara, USA) was used as the excitation source at a power output of $6 \mathrm{~mW}$. The surface chemical states/electronic properties of the carbon monoliths annealed at 900 and $1250{ }^{\circ} \mathrm{C}$ were characterised by X-ray photoelectron spectroscopy (XPS), using a Kratos AXIS-165 electron spectrometer with monochromatic Al $\mathrm{K} \alpha(1486.6 \mathrm{eV}) \mathrm{X}$-ray source, with a chamber pressure of $10^{-9}$ mbar. Thermogravimetric analysis was performed on a Labsys Evo (Setaram, Caluire, France) instrument

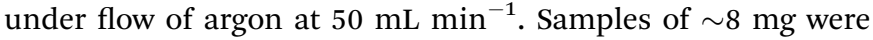
placed in alumina crucibles for analysis. A heating rate of $2{ }^{\circ} \mathrm{C}$ $\min ^{-1}$ from 30 to $900{ }^{\circ} \mathrm{C}$ was used, followed by a hold period of 2 
hours at $900{ }^{\circ} \mathrm{C}$. The temperature was then ramped from 900 to $1100{ }^{\circ} \mathrm{C}$ at a rate of $2{ }^{\circ} \mathrm{C} \mathrm{min}^{-1}$ in order to closely replicate the pyrolysis temperature ramp conditions. A slower heating rate was employed to avoid the sudden evolution of gaseous products, which could affect the precision balance in the instrument.

\section{Results and discussion}

\section{Synthesis of porous graphitic carbon monoliths with} thermally annealed carbon onions

It is known that the partial graphitisation of $\mathrm{RF}$ resin can be achieved through the addition of a catalyst, which reduces the temperature needed to achieve graphitisation, and also results in the formation of a mesoporous structure. ${ }^{24,25} \mathrm{Here}, \mathrm{FeCl}_{3}$ was added as the graphitisation catalyst and to produce the desired mesoporous network. In this work, it was expected that the addition of DND should similarly promote localised graphitisation, due in part to its high thermal conductivity, and also its own graphitisation and transformation into carbon onion nano-structures.

Fig. 2(a) shows a low magnification SEM image of a cross section of the precursor rod containing DND prior to undergoing carbonisation. The surface area of the uncarbonised material determined using nitrogen adsorption measurements was a low $55 \mathrm{~m}^{2} \mathrm{~g}^{-1}$, with a pore volume of $0.21 \mathrm{~cm}^{3} \mathrm{~g}^{-1}$, and an average pore diameter of $15 \mathrm{~nm}$. The isotherm displayed a limiting step after the hysteresis loop suggesting that pores were completely filled during the analysis.

In prior reports on the formation of similar carbon monoliths, thermogravimetric analysis (TGA) has been used to observe the various phases of carbonisation and graphitisation. ${ }^{11,14}$ Here the impact of DND inclusion on these processes was also observed using TGA, under conditions similar to those used in the normal carbonisation process, although here under argon flow. The TGA curve for DND itself (Fig. 2(b)) shows an initial weight loss of $\sim 7 \%$ between $30-160{ }^{\circ} \mathrm{C}$, which is due to water losses. DND is known to be particularly hygroscopic and can contain multiple layers of water molecules on its surface, including those bound directly to the carbon surface or to the negatively charged surface functional groups. Further layers may also be bound through hydrogen bonds or other noncovalent interactions. ${ }^{26}$ Following this initial weight loss, the TGA curve shows an unusual slight drift upwards between 200 and $350{ }^{\circ} \mathrm{C}$, followed by a steady decline in weight between $400-$ $900{ }^{\circ} \mathrm{C}$, which is likely due to the removal of surface organic groups ${ }^{27}$ and the loss of some elemental impurities, which can make up a significant amount of the DND mass $\left(13.8 \mathrm{mg} \mathrm{g}^{-1}\right.$ with large contributions from $\mathrm{B}, \mathrm{Na}, \mathrm{Si}, \mathrm{Ca}$, and $\mathrm{Fe}) \cdot{ }^{28,29}$ It is known that the loss of oxygen-containing groups occurs below $900{ }^{\circ} \mathrm{C}$ in an inert atmosphere, and of $\mathrm{CH}_{x}$ groups between 900$1150{ }^{\circ} \mathrm{C} . .^{30,31}$ Above $\sim 700{ }^{\circ} \mathrm{C}$ the graphitisation of the DND normally begins. A total weight loss of $33.7 \%$ occurred between $30-$ $1100^{\circ} \mathrm{C}$. It has been shown that DND evolves large quantities of $\mathrm{CO}_{2}$ and $\mathrm{CO}$ during thermal annealing. ${ }^{32}$

During thermal annealing, the DND will become graphitised, as the $\mathrm{sp}^{3}$ hybrid is less energetically stable than the $\mathrm{sp}^{2}$, and graphite is a more stable phase. Graphitisation occurs in a layer-by-layer fashion, from the more reactive surface regions inwards. Xu et al. have studied the thermal annealing of DND in an inert atmosphere, showing that the DND undergoes graphitisation between $670-1100{ }^{\circ} \mathrm{C} .{ }^{33,34}$ As seen in Fig. 2(b), the precursor rod containing DND undergoes $8 \%$ weight loss below $200{ }^{\circ} \mathrm{C}$. At lower temperatures these losses can be accounted for as the evolution of water, excess phenol or other low molecular weight compounds, as well as the release of $\mathrm{CO}, \mathrm{CO}_{2}, \mathrm{H}_{2}$ and other gases. ${ }^{35}$ During the pyrolysis process, the $\mathrm{RF}$ resin undergoes further weight losses, physical shrinkage and pore formation as the condensation of the polymer structure takes place, and the resin is gradually converted to amorphous carbon. ${ }^{36}$ The total weight loss for the composite material between $30-1100{ }^{\circ} \mathrm{C}$ was $26.2 \%$, which is very similar to the total
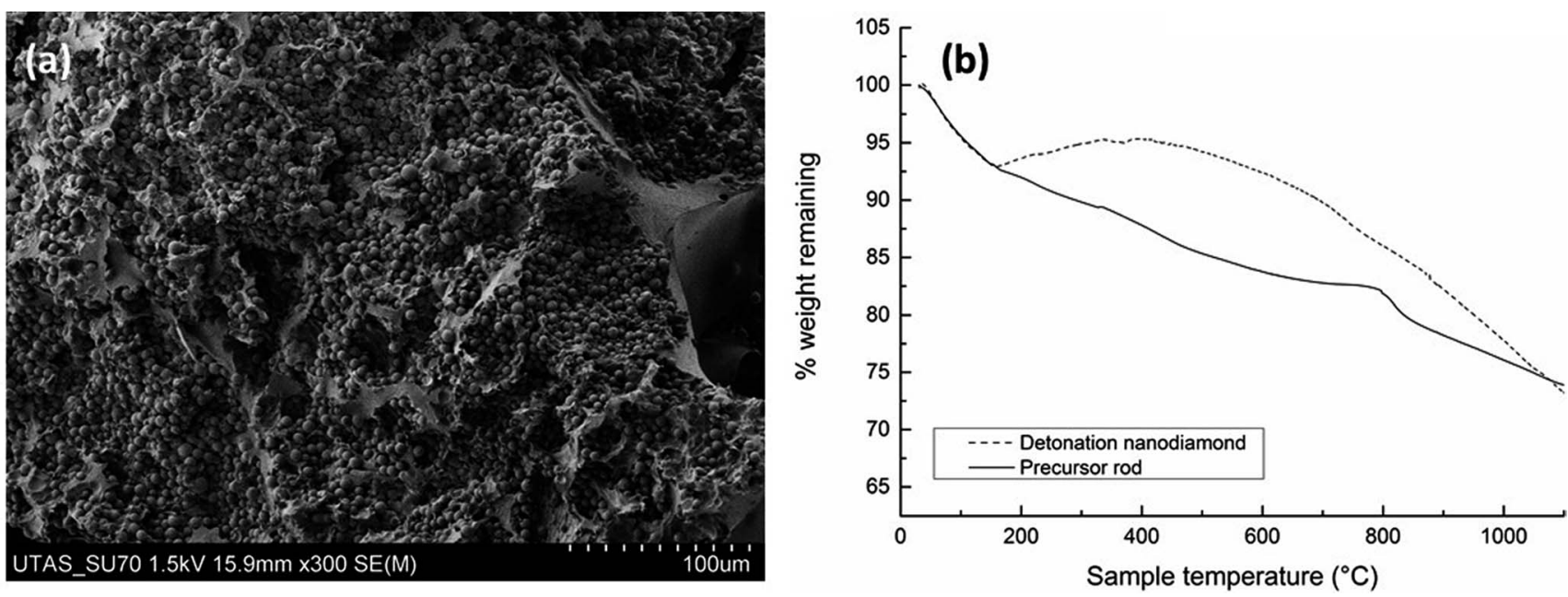

Fig. 2 (a) SEM image taken of a cross section of the precursor rod containing silica particles, and detonation nanodiamond (DND), before carbonisation at $1250^{\circ} \mathrm{C}$. (b) Thermogravimetric analysis curves for DND, and precursor rod containing the resorcinol-formaldehyde resin, with $\mathrm{Fe}^{3+}$ catalyst, silica particles, and DND. 


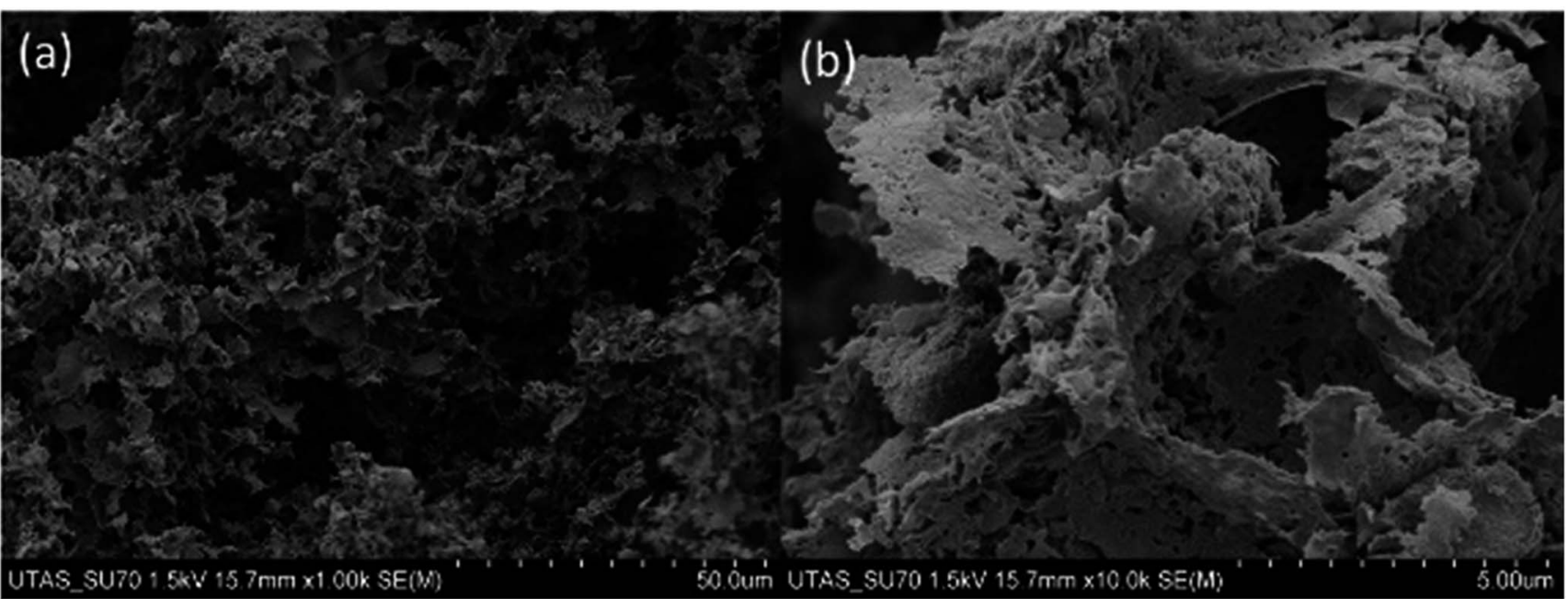

Fig. 3 SEM images showing the porous graphitic carbon monolith with carbon onions. (a) Macroporous network mag X $1 \mathrm{~K}$, (b) mag $\mathrm{X} 10 \mathrm{~K}$.

weight loss $(25 \%)$ observed for carbonisation of a composite monolith prepared with an RF precursor rod containing an $\mathrm{Fe}^{3+}$ catalyst, and a $\mathrm{C}_{60}$-modified silica template. ${ }^{16}$ However, these losses are both lower than those reported for a similar RF resin, prepared without the addition of any nano-carbons.

\section{Structure and morphology}

The porous carbon composite materials prepared exhibited a bimodal porous structure, where both macropores and mesopores were clearly evident. The interconnected macroporous network is visible in Fig. 3(a). The $\sim 5 \mu \mathrm{m}$ diameter pores result from the densely packed silica template that was removed during the HF treatment. The presence of larger voids within the macroporous network was noted for the DND-containing composite materials, which were less obvious for the bare carbon monolithic material that was prepared for comparative purposes. The addition of an aqueous suspension of DND may explain the appearance of such voids in the composite material, since 1-butanol has limited solubility in water. An increased level of porosity (compared to the bare monolithic carbon) resulted from the presence of DND, and the walls of the macropores were notably thinner, more fragile and irregular, as the DND content of the composite physically disrupts complete polymerisation of the resorcinol resin (Fig. 3(b)).
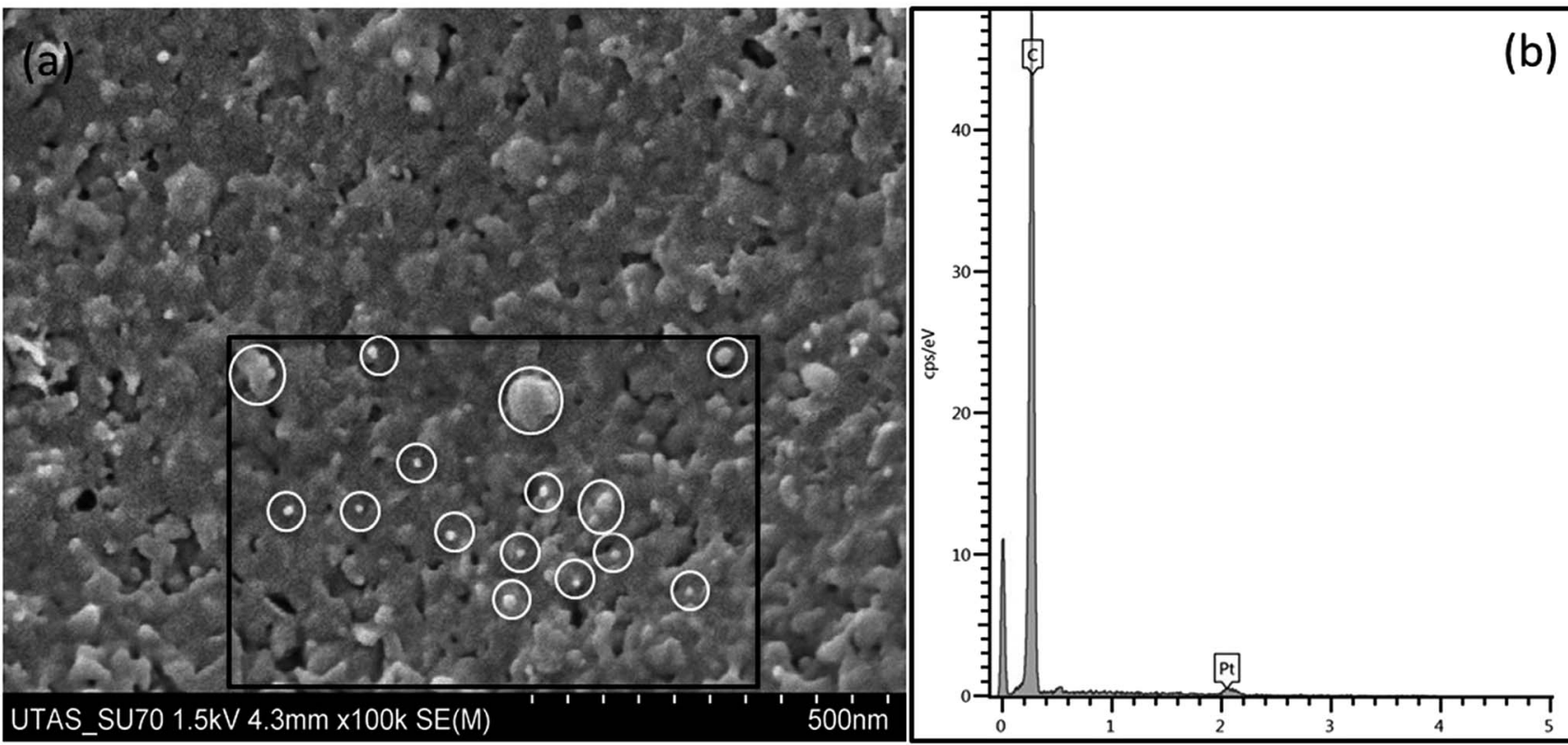

Fig. 4 (a) High magnification $(\times 100 \mathrm{~K}$ ) image of the wall of monolith macropore, showing the presence of a mesoporous structure (with surviving surface detonation nanodiamonds highlighted between 10 and $100 \mathrm{~nm}$ ). (b) EDX spectrum for carbon monolith showing presence of carbon only (Pt from sputter coating). 
Closer examination of the wall structure of the macropores revealed smooth pore walls with a low instance of raised surface features visible by low magnification SEM imaging. These regions showing irregular raised porous surfaces may be linked to some infiltration of the precursor polymerisation solution in to the $55 \mathrm{~nm}$ pores of the silica template. This effect is highly dependent on wetting of the silica particles, as well as the viscosity of the precursor solution. An SEM image showing such areas with irregular surface morphology is shown in ESI Fig. S1. $\dagger$ Furthermore, SEM imaging was performed on cross sections of the uncarbonised monolith precursor and has revealed some instances of accumulation of polymerised resin on the surface of silica beads (highlighted in ESI Fig. S2 $\dagger$ ). Abundant mesopores were also visible by SEM imaging, and this structure was similar for materials annealed at both 900 and $1250{ }^{\circ} \mathrm{C}$. Examination of monoliths graphitised at the lower temperatures also revealed the presence of surviving DND embedded within the mesoporous walls and attached upon the surface, both as single digit DND $(<10 \mathrm{~nm})$ and clusters of up to $100 \mathrm{~nm}$ size (see Fig. 4(a)). Surface EDX analysis was performed on the macroporore walls of each of the composite materials, confirming the carbon purity of the composite throughout (Fig. 4(b)). Platinum was detected due to the thin layer of platinum coating the samples to facilitate imaging by SEM. Negligible amounts of Si were also detected in some regions of the macropore walls, although there was no evidence of the $\mathrm{Fe}^{3+}$ catalyst remaining.

Comparison of the carbon monoliths annealed at 900 and $1250{ }^{\circ} \mathrm{C}$ using TEM revealed the graphitic nature of the monolith, with localised graphitic structures visible. The RF resin does not undergo full graphitisation, as evidenced by the regions of amorphous carbon also visible within the TEM images. However, the thermal conversion of the DND to carbon onions within the $1250{ }^{\circ} \mathrm{C}$ monolith was confirmed. Within this sample no obvious evidence of untransformed DND particles could be seen. These TEM images are shown within Fig. 5. It has previously been shown that carbon onions begin to form in the range of $900-1100{ }^{\circ} \mathrm{C}$ under a low vacuum, ${ }^{37}$ and in the range of 1100-1200 ${ }^{\circ} \mathrm{C}$ in an inert atmosphere. ${ }^{38}$ In this case the one hour hold period at $1250{ }^{\circ} \mathrm{C}$ during pyrolysis was sufficient to quantitatively transform the DND. The carbon onions resulting from the thermal annealing of DND within the monolithic composite are shown in Fig. 5(b). The onions were quasispherical in shape, and they had an average diameter of 5.24 $\mathrm{nm}( \pm 0.85 \mathrm{~nm})$. The average interlayer spacing between the graphitic shells is $3.32 \AA$, which is between values previously reported for onions with five (3.35 $\AA$ ) to ten $(3.24 \AA)$ shells. ${ }^{38}$

TEM analysis confirmed that by reducing the maximum temperature of pyrolysis to $900{ }^{\circ} \mathrm{C}$, the DND structures could actually be preserved within the composite material. Fig. 5(c) shows a cluster of intact DND structures present within the final composite material. The DNDs shown were not graphitised at $900{ }^{\circ} \mathrm{C}$ and do not show evidence of the graphitisation of their outer layers. It is likely their structure was preserved at this temperature as a result of their being embedded within the carbon monolithic rod. The lattice fringes corresponding to the (111) planes of diamond are clearly visible, and the average

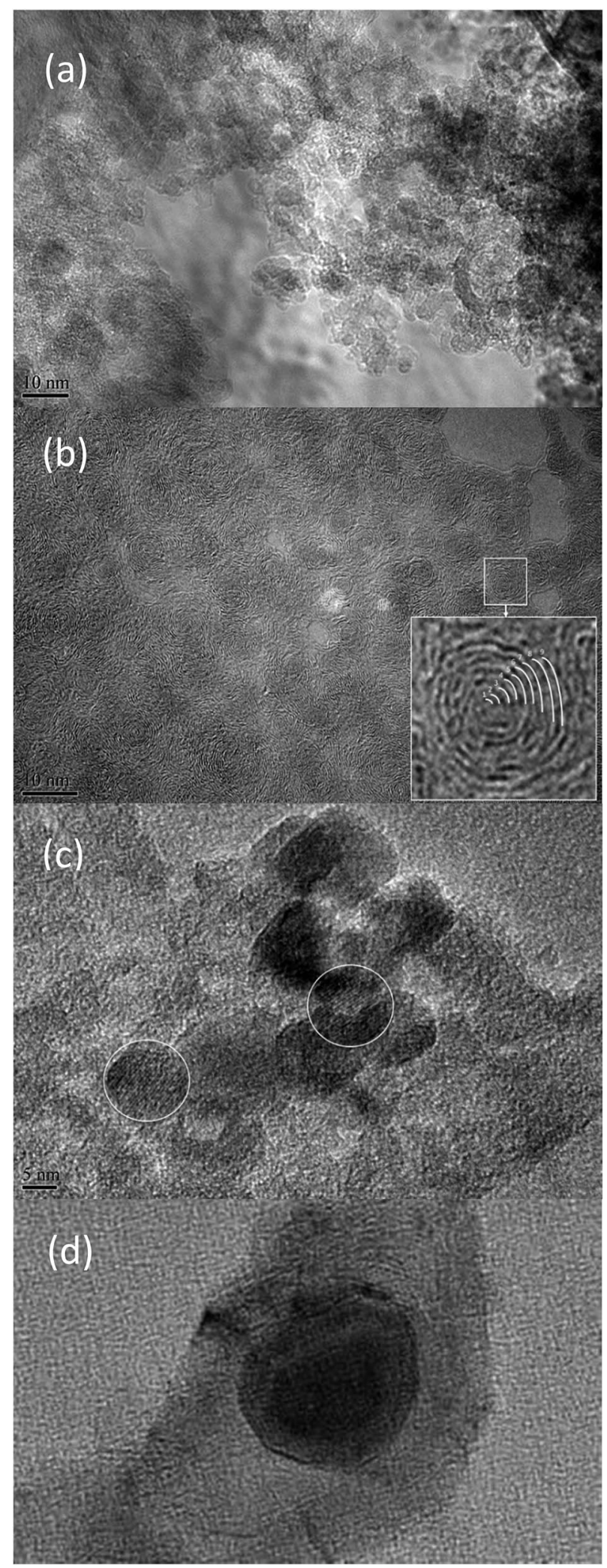

Fig. 5 TEM images of porous graphitic carbon composites. (a) Amorphous carbon, (b) carbon onions resulting from the thermal annealing of detonation nanodiamond (DND) under $\mathrm{N}_{2}$ at $1250{ }^{\circ} \mathrm{C}$, (c) DND remains intact within the porous graphitic carbon composite following pyrolysis up to $900{ }^{\circ} \mathrm{C}$, (d) DND showing surface graphitisation following pyrolysis up to $900^{\circ} \mathrm{C}$. 
interlayer spacing measured on the DND within the composite was $2.06 \pm 0.28 \AA$. The average particle size as observed by TEM was $11.4 \pm 0.9 \mathrm{~nm}$, as expected based upon the specification of the commercial sample (5-15 nm particle diameter). However, some DND particles did show partial graphitisation at $900{ }^{\circ} \mathrm{C}$, which agrees with work by Cebik et al., which demonstrated that annealing DND in an inert atmosphere at $900-1000{ }^{\circ} \mathrm{C}$ can lead to the conversion of some of the surfaces of ND to layered $\mathrm{sp}^{2}$ carbon. ${ }^{39}$ DND present at the surface level of the composite material would not experience the same environment as those particles which were embedded in the rod during pyrolysis at $900{ }^{\circ} \mathrm{C}$, and so partial layer-by-layer conversion to $\mathrm{sp}^{2}$ carbon has occurred, as visible in Fig. 5(d). Thus, careful control of temperature of pyrolysis allows control of the nature of the nano-carbons present in the final monolithic composite materials.

Raman spectroscopy was applied to shed further light on the structure of the monolithic composite materials. The disorder in graphite can give rise to several characteristic Raman peaks. ${ }^{40}$ The 800-2000 $\mathrm{cm}^{-1}$ region in the Raman spectrum shows common features for all carbons. The $\mathrm{G}$ and $\mathrm{D}$ peaks are typically around 1560 and $1360 \mathrm{~cm}^{-1}$ respectively, for visible

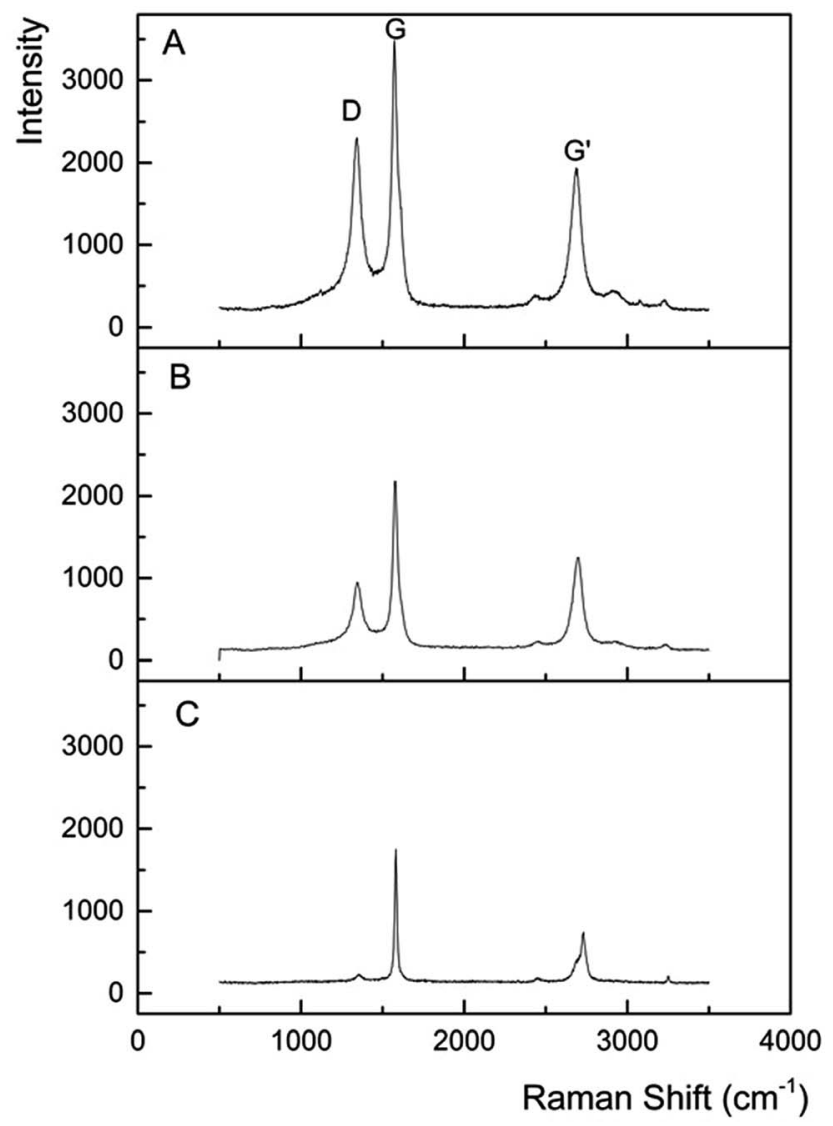

Fig. 6 Raman spectra for A - bare carbon monolith (prepared without addition of carbon nanoparticles for comparative purposes), B carbon on carbon monolithic composite, and C - commercial graphite. Both carbon monolithic materials underwent pyrolysis at $1250^{\circ} \mathrm{C}$ excitation. ${ }^{41}$ The spectrum for the blank porous carbon monolith is also shown in Fig. 6(a). Three peaks were observed in the Raman spectrum for the monolithic composites containing carbon onions (Fig. 6(b)). These three peaks are usually seen for carbonaceous materials with both $\mathrm{sp}^{2}$ and $\mathrm{sp}^{3}$ bonds present. The $\mathrm{G}$ band appears at $\sim 1580 \mathrm{~cm}^{-1}$ and corresponds to the $\mathrm{E}_{2 \mathrm{~g}}$ optical mode in a two-dimensional network structure, always seen for $\mathrm{sp}^{2}$ carbon materials. The $\mathrm{D}$ band is visible at $\sim 1335$ $\mathrm{cm}^{-1}$ and it is associated with disordered carbon, ${ }^{42}$ denoting a loss of hexagonal symmetry in the material (for highly ordered pyrolytic graphite, this peak is very small or even negligible, see Fig. 6(c) for comparison). The intensity of the $\mathrm{D}$ band to the $\mathrm{G}$ band $\left(R=I_{\mathrm{D}} / I_{\mathrm{G}}\right)$ can be used to illustrate the degree of graphitisation in a material. Here, the blank carbon monolithic material showed an $R$-value of 0.64 , and the $1250{ }^{\circ} \mathrm{C}$ formed composite monolith gave a value of 0.37 (the $R$-value for commercial graphite was 0.14 ), thus confirming the greater graphitic nature of the carbon on carbon composite material. This supports the proposal that inclusion of the DND promotes localised graphitisation, both due to its inherent high thermal conductivity, and self-graphitisation during pyrolysis.

Temperature of pyrolysis is an important factor to consider here, as the composite resin remains a predominantly disordered material following its carbonisation up to $1250{ }^{\circ} \mathrm{C}$. The $\mathrm{G}^{\prime}$ band is seen to appear at $\sim 2680 \mathrm{~cm}^{-1}$ and typically appears for $\mathrm{sp}^{2}$ carbon materials resulting from a second order two phonon process. ${ }^{43}$ These results confirm the presence of both graphitic and amorphous carbon in the carbon framework of the monolithic composites. Analysis by XPS further demonstrated the effect of pyrolysis temperature on partial graphitisation of the composites (Fig. 7). A downshift in the binding energy was observed for the carbon core level (C 1s) spectral comparison of monoliths carbonised at $900{ }^{\circ} \mathrm{C}(285.5 \pm 0.05 \mathrm{eV})$ and $1250{ }^{\circ} \mathrm{C}$ $(284.5 \pm 0.05 \mathrm{eV})$ under a nitrogen atmosphere. This observation was similar to that reported by Xie et al. for the annealing of DND at 900 and $1500{ }^{\circ} \mathrm{C},{ }^{\mathbf{4 4}}$ and within studies by Krishnamurthy and co-workers. ${ }^{23,45}$ This shift in binding energy relates to the

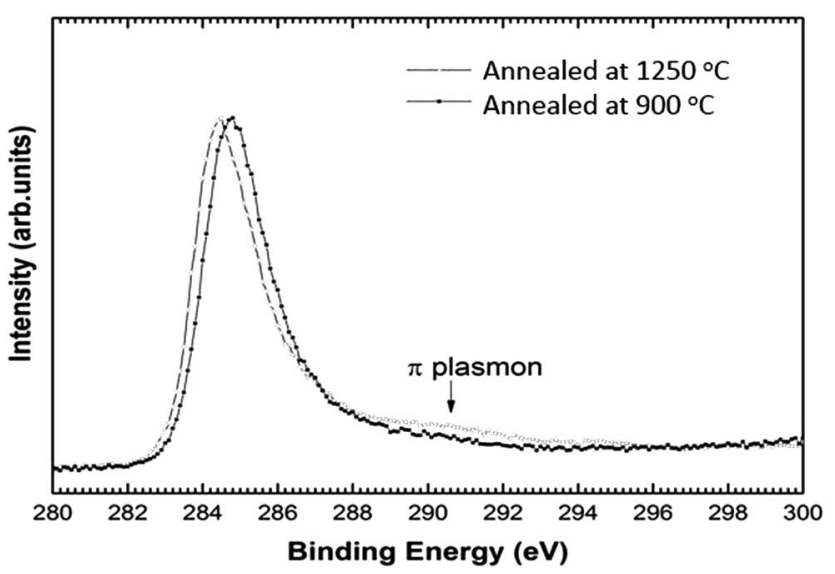

Fig. 7 C 1s XPS spectra of carbon monoliths containing detonation nanodiamond which underwent pyrolysis at temperatures of $900{ }^{\circ} \mathrm{C}$ and $1250^{\circ} \mathrm{C}$. Spectra were obtained in normal emission geometry at a photon energy of $1486.6 \mathrm{eV}$. 
material moving towards graphitisation as a function of the pyrolysis temperature. The binding energy of $284.5 \mathrm{eV}$ for the composite that underwent pyrolysis at $1250{ }^{\circ} \mathrm{C}$ is slightly higher than one for graphite $(284.4 \mathrm{eV}) .{ }^{30}$ Also evident in the $1250{ }^{\circ} \mathrm{C}$ annealed sample, but not featured within the $900{ }^{\circ} \mathrm{C}$ sample, was the shake-up feature related to the $\pi$ to $\pi^{*}$ transition at around $290.8 \pm 0.05 \mathrm{eV}$, commonly seen in more graphitised materials.

It is widely known that DND typically has a high concentration of structural defects on its surface, which increases the surface reactivity. Pyrolysis of the composites at $900{ }^{\circ} \mathrm{C}$ produced some hybrid nanocarbons combining the core properties of $\mathrm{ND}$, with the surface reactivity of $\mathrm{sp}^{2}$-based nanocarbons, as discussed above in relation to Fig. 5(d). ${ }^{46}$ The production of hybrid nanocarbons is similar in effect to previous work reported by Ostrovidova et al. where ND particles were bonded by a graphite-like matrix in order to produce a high surface area porous nanodiamond composite pressmoulded tablet for immobilisation of biomolecules. ${ }^{47}$

\section{High-surface area carbon monoliths}

The adsorption of nitrogen on the blank carbon monolith showed a type IV isotherm, which is typical of mesoporous materials (Fig. 8(A)). Similarly, the carbon composite monolith (carbonised up to $1250{ }^{\circ} \mathrm{C}$ ) also exhibited a type IV isotherm (Fig. 8(B)). The initial region of the isotherm where an increase in adsorption followed by the knee is the point at which monolayer adsorption is preceded by multilayer adsorption. The presence of the hysteresis loop is indicative of capillary condensation within the mesopores. The hysteresis loop is type $\mathrm{H} 3$, which is associated with the presence of slit-like pores, and the limiting step (at high relative pressure) seen for many mesoporous sorbents of isotherm type IV is not present in a type $\mathrm{H} 3$ hysteresis loop. This suggests that complete pore filling may not have occurred. ${ }^{48}$ The material does not show a sharp condensation/evaporation step, which typically characterises a

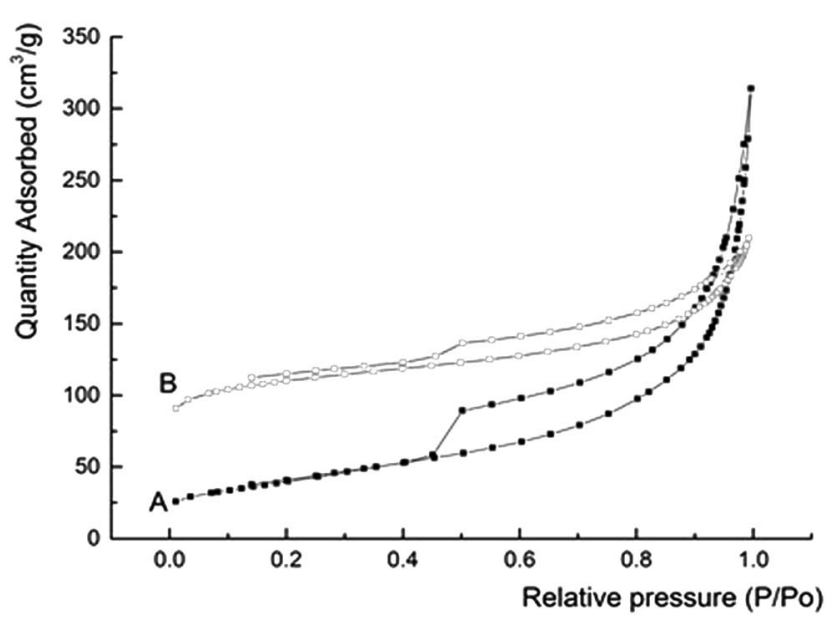

Fig. 8 Nitrogen adsorption-desorption isotherms for A - bare carbon monolith (prepared without the addition of carbon nanoparticles for comparative purposes) and B - carbon on carbon monolithic composite containing carbon onions.
Table 1 Structural characteristics of carbon monolithic composites

\begin{tabular}{llll}
\hline Monolith & $\begin{array}{l}S_{\mathrm{BET}}{ }^{a} \\
\left(\mathrm{~m}^{2} \mathrm{~g}^{-1}\right)\end{array}$ & $\begin{array}{l}V_{\mathrm{p}}{ }^{a} \\
\left(\mathrm{~cm}^{3} \mathrm{~g}^{-1}\right)\end{array}$ & $\begin{array}{l}\text { Pore } \\
\text { diameter }^{b}(\mathrm{~nm})\end{array}$ \\
\hline $\begin{array}{llll}\text { Bare carbon monolith } \\
\text { Carbon monolith }\end{array}$ & $115 \pm 8$ & $0.33 \pm 0.05$ & $11.7 \pm 0.8$ \\
& $214 \pm 17$ & $0.35 \pm 0.04$ & $10.5 \pm 3.9$
\end{tabular}

with carbon onions

${ }^{a}$ The Brunauer-Emmett-Teller (BET) method was used to calculate specific surface areas. ${ }^{b}$ The Barrett-Joyner-Halenda (BJH) method was used to calculate mesopore diameters from the adsorption branch of the isotherm.

narrow pore size distribution. Therefore, these materials likely exhibit a wide mesopore size distribution with irregular pore shapes. The steep desorption region in the hysteresis loop is associated with the (forced) closure of the loop due to the socalled tensile strength effect. Closure of the hysteresis loop at $P / P_{0} \sim 0.4$ indicates that the mesopores were relatively small in size. Estimated mesopore diameters for both materials were in agreement, with the Barrett-Joyner-Halenda (BJH) method applied $^{49}$ to find mesopore diameters of $11.7 \pm 0.8 \mathrm{~nm}$ and 10.5 $\pm 3.9 \mathrm{~nm}$, for the blank carbon monolith and monolithic composite, respectively (see Table 1). The carbon monolithic composite had a slightly greater mesopore volume of $0.35 \pm$ $0.04 \mathrm{~cm}^{3} \mathrm{~g}^{-1}$ compared to $0.33 \pm 0.05 \mathrm{~cm}^{3} \mathrm{~g}^{-1}$ for the blank monolith. The addition of DND appears to have increased the prevalence of mesopores with smaller diameters. Significantly then it is clear that the addition of DND to the carbon monolith can be used to affect both the macroporous and mesoporous structure, as evidenced by both BET, and SEM images discussed previously (Fig. 3). The average BET surface area calculated for the monolithic composite at $P / P_{0}$ from 0.05 to 0.30 was $214 \mathrm{~m}^{2}$ $\mathrm{g}^{-1}$, nearly twice which obtained for the bare carbon monolith, which was $115 \mathrm{~m}^{2} \mathrm{~g}^{-1}$. The higher surface area in the composite material is due to the increased macro and meso-porosity, and from the surface area contribution from the nano-carbon materials embedded in the carbon skeleton, which are known to display high surface areas. ${ }^{50}$ This represents a significant increase, which if related to the concentration of DND added, could provide a unique method for control of this important parameter.

\section{Conclusions}

New bimodal carbon on carbon monolithic composites were successfully prepared by embedding DND in a resorcinol formaldehyde precursor mixture, containing $\mathrm{Fe}^{3+}$ as a catalyst for localised graphitisation, and silica gel as a hard template. Pyrolysis cycles reached a maximum temperature of $1250{ }^{\circ} \mathrm{C}$, which was sufficiently high enough to result in the full graphitisation of the DND precursor, forming quasi-spherical carbon onions within the monolith, which had an average diameter of $5.24 \mathrm{~nm}$. The inclusion of DND increased the graphitisation of the composite material, which contained both $\mathrm{sp}^{2}$ and $\mathrm{sp}^{3}$ carbon phases following pyrolysis. Both the 
macroporous network and mesopores were affected by the addition of DND, and the BET surface area and pore volume were increased in comparison with a blank carbon monolith. It was also shown that the DND could be preserved within the composite by reducing the temperature of pyrolysis, thus demonstrating the ability to easily control nano-carbon synthesis within a monolithic composite. Carbon monolithic composites with carbon onions are suitable for application in a number of areas such as electrode materials, chromatographic applications and extraction processes for larger molecules including organic pollutants or biomolecules.

To the best of the authors' knowledge, this is the first report of the controlled production of carbon onions from DND within such a carbon monolithic composite. It demonstrates that the type of nano-carbons present in the final monolithic composite can be tuned, simply by controlling the temperature of pyrolysis.

\section{Acknowledgements}

The authors thank the Australian Research Council (Discovery grant DP110102046) for research funding. We would like to thank Mrs Heather Davies, Dr Brendan Twamley, Dr Karsten Goemann and Dr Sandrin Feig for their technical support and assistance with electron microscopy imaging.

\section{Notes and references}

1 P. Colombo, Science, 2008, 322, 381-383.

2 D.-W. Wang, F. Li, M. Liu, G. Q. Lu and H.-M. Cheng, Angew. Chem., Int. Ed., 2008, 47, 373-376.

3 F. U. Ruo-Wen, L. I. Zheng-Hui, L. Ye-Ru, L. I. Feng, X. U. Fei and W. U. Ding-Cai, New Carbon Mater., 2011, 26, 171-179.

4 M. Sevilla and A. B. Fuertes, Energy Environ. Sci., 2011, 4, 1765-1771.

5 G.-P. Hao, W.-C. Li, D. Qian and A.-H. Lu, Adv. Mater., 2010, 22, 853-857.

6 V. K. Gupta and T. A. Saleh, Environ. Sci. Pollut. Res., 2013, 20, 2828-2843.

7 L. Chuenchom, R. Kraehnert and B. M. Smarsly, Soft Matter, 2012, 8, 10801-10812.

8 A. D. Roberts, X. Li and H. Zhang, Chem. Soc. Rev., 2014, 43, 4341-4356.

9 S. Dutta, A. Bhaumik and K. C.-W. Wu, Energy Environ. Sci., 2014, 7, 3574-3592.

10 C. Liang, S. Dai and G. Guiochon, Anal. Chem., 2003, 75, 4904-4912.

11 A. H. Eltmimi, L. Barron, A. Rafferty, J. P. Hanrahan, O. Fedyanina, E. Nesterenko, P. N. Nesterenko and B. Paull, J. Sep. Sci., 2010, 33, 1231-1243.

12 V. Ruiz, C. Blanco, R. Santamaría, J. M. Ramos-Fernández, M. Martínez-Escandell, A. Sepúlveda-Escribano and F. Rodríguez-Reinoso, Carbon, 2009, 47, 195-200.

13 X. He, K. B. Male, P. N. Nesterenko, D. Brabazon, B. Paull and J. H. T. Luong, ACS Appl. Mater. Interfaces, 2013, 5, 8796-8804.

14 Z. Wang, F. Li, N. S. Ergang and A. Stein, Chem. Mater., 2006, 18, 5543-5553.
15 L. Qiu, J. Z. Liu, S. L. Y. Chang, Y. Wu and D. Li, Nat. Commun., 2012, 3, 1241-1247.

16 X. He, E. P. Nesterenko, P. N. Nesterenko, D. Brabazon, L. Zhou, J. D. Glennon, J. H. T. Luong and B. Paull, ACS Appl. Mater. Interfaces, 2013, 5, 8572-8580.

17 M. S. Mauter and M. Elimelech, Environ. Sci. Technol., 2008, 42, 5843-5859.

18 M. B. Seymour, C. Su, Y. Gao, Y. Lu and Y. Li, J. Nanopart. Res., 2012, 14, 1087.

19 E. K. Chow, X.-Q. Zhang, M. Chen, R. Lam, E. Robinson, H. Huang, D. Schaffer, E. Osawa, A. Goga and D. Ho, Sci. Transl. Med., 2011, 3, 73ra21.

20 T. J. Merkel and J. M. DeSimone, Sci. Transl. Med., 2011, 3, 73 ps8.

21 V. L. Kuznetsov, A. L. Chuvilin, Y. V. Butenko, I. Y. Mal'kov and V. M. Titov, Chem. Phys. Lett., 1994, 222, 343-348.

22 D. Pech, M. Brunet, H. Durou, P. Huang, V. Mochalin, Y. Gogotsi, P.-L. Taberna and P. Simon, Nat. Nanotechnol., 2010, 5, 651-654.

23 Y. V. Butenko, S. Krishnamurthy, A. K. Chakraborty, V. L. Kuznetsov, V. R. Dhanak, M. R. C. Hunt and L. Šiller, Phys. Rev. B: Condens. Matter Mater. Phys., 2005, 71, 075420.

24 A. Oya and H. J. Marsh, J. Mater. Sci., 1982, 17, 309-322. 25 M. Sevilla and A. B. Fuertes, Carbon, 2006, 44, 468-474.

26 S. S. Batsanov, E. V. Lesnikov, D. A. Dan'kin and D. M. Balakhanov, Appl. Phys. Lett., 2014, 104, 133105.

27 W.-W. Zheng, Y.-H. Hsieh, Y.-C. Chiu, S.-J. Cai, C.-L. Cheng and C. Chen, J. Mater. Chem., 2009, 19, 8432-8441.

28 D. P. Mitev, A. T. Townsend, B. Paull and P. N. Nesterenko, J. Mater. Sci., 2014, 49, 3573-3591.

29 D. P. Mitev, A. T. Townsend, B. Paull and P. N. Nesterenko, Carbon, 2013, 60, 326-334.

30 Y. V. Butenko, V. L. Kuznetsov, E. A. Paukshtis, A. I. Stadnichenko, I. N. Mazov, S. I. Moseenkov, A. I. Boronin and S. V. Kosheev, Fullerenes, Nanotubes, Carbon Nanostruct., 2006, 14, 557-564.

31 V. Mochalin, A. Osswald and Y. Gogotsi, Chem. Mater., 2009, 21, 273-279.

32 K. W. Lin, C. L. Cheng and H. C. Chang, Chem. Mater., 1998, 10, 1735-1737.

33 N. S. Xu, J. Chen and S. Z. Deng, Diamond Relat. Mater., 2002, 11, 249-256.

34 J. Chen, S. Z. Deng, J. Chen, Z. X. Yu and N. S. Xu, Appl. Phys. Lett., 1999, 74, 3651-3653.

35 T. H. Ko, W. S. Kuo and Y. R. Lu, Polym. Compos., 2000, 21, 96-103.

36 T. H. Ko, W. S. Kuo and Y. H. Chang, J. Appl. Polym. Sci., 2001, 81, 1084-1089.

37 Q. Zou, M. Wang, Y. Li, Y. Zhao and L. Zou, Sci. China, Ser. E: Technol. Sci., 2009, 52, 3683-3689.

38 Z. Qiao, J. Li, N. Zhao, C. Shi and P. Nash, Scr. Mater., 2006, 54, 225-229.

39 J. Cebik, J. K. McDonough, F. Peerally, R. Medrano, I. Neitzel, Y. Gogotsi and S. Osswald, Nanotechnology, 2013, 24, 205703.

40 F. Tuinstra and J. L. Koenig, J. Chem. Phys., 1970, 53, 11261130. 
41 A. C. Ferrari and J. Robertson, Philos. Trans. R. Soc. London, Ser. A, 2004, 362, 2477-2512.

42 A. C. Ferrari and J. Robertson, Phys. Rev. B: Condens. Matter Mater. Phys., 2000, 61, 14095-14107.

43 X. He, L. Zhou, E. P. Nesterenko, P. N. Nesterenko, B. Paull, J. O. Omamogho, J. D. Glennon and J. H. Luong, Anal. Chem., 2012, 84, 2351-2357.

44 F. Y. Xie, W. G. Xie, L. Gong, W. H. Zhang, S. H. Chen, Q. Z. Zhang and J. Chen, Surf. Interface Anal., 2010, 42, 1514-1518.

45 S. Krishnamurthy, Y. V. Butenko, V. R. Dhanak, M. R. C. Hunt and L. Šiller, Carbon, 2013, 52, 145-149.
46 T. Petit, J.-C. Arnault, H. A. Girard, M. Sennour and P. Bergonzo, Phys. Rev. B: Condens. Matter Mater. Phys., 2011, 84, 233407.

47 G. U. Ostrovidova, A. V. Makeev, A. V. Biryukov and S. K. Gordeev, Mater. Sci. Eng., 2003, 23, 377-381.

48 S. Lowell, J. E. Shields, M. A. Thomas and M. Thommes, in Characterization of Porous Solids and Powders: Surface Area, Pore Size and Density, Kluwer Academic Publishers, London, 2004.

49 E. P. Barrett, L. G. Joyner and P. P. Halenda, J. Am. Chem. Soc., 1951, 73, 373-380.

50 J. K. McDonough and Y. Gogotsi, Electrochem. Soc. Interface, 2013, 3, 61-66. 